Q.-S. Chi, L. Fernández and H. Wu

Nagoya Math. J.

Vol. 156 (1999), 187-214

\title{
NORMALIZED POTENTIALS OF MINIMAL SURFACES IN SPHERES
}

\author{
QUO-SHIN CHI , LUIS FERNÁNDEZ AND HONGYOU WU
}

\begin{abstract}
We determine explicitly the normalized potential, a Weierstrasstype representation, of a superconformal surface in an even-dimensional sphere $S^{2 n}$ in terms of certain normal curvatures of the surface. When the Hopf differential is zero the potential embodies a system of first order equations governing the directrix curve of a superminimal surface in the twistor space of the sphere. We construct a birational map from the twistor space of $S^{2 n}$ into $\mathbb{C} P^{n(n+1) / 2}$. In general, birational geometry does not preserve the degree of an algebraic curve. However, we prove that the birational map preserves the degree, up to a factor 2, of the twistor lift of a superminimal surface in $S^{6}$ as long as the surface does not pass through the north pole. Our approach, which is algebrogeometric in nature, accounts in a rather simple way for the aforementioned first order equations, and as a consequence for the particularly interesting class of superminimal almost complex curves in $S^{6}$. It also yields, in a constructive way, that a generic superminimal surface in $S^{6}$ is not almost complex and can achieve, by the above degree property, arbitrarily large area.
\end{abstract}

\section{$\S$ 0. Introduction}

An important tool for studying minimal surfaces is the Weierstrass representation ([25]) which, instead of dealing directly with the surface itself, describes the surface via certain holomorphic data associated with it. The recent advances in the theory of minimal surfaces in $\mathbb{R}^{3}$ is a triumphant example of this principle ([19],[20],[26]).

Recently, a Weierstrass-type representation, referred to as the normalized potential by the authors ([12]), has been proven to exist through the loop-group analysis for any harmonic map from a Riemann surface into a compact symmetric space. The particular case of harmonic maps into spheres has been under extensive investigations in the past several decades ([2],[3],[7],[8],[14],[18],[22],[23], to say the least).

Recall that a superconformal (or cyclic) surface in $S^{n}$ is one whose harmonic sequence is cyclic of order $n$. On the other hand, a supermini-

Received May 7, 1997. 
mal surface is one whose harmonic sequence degenerates to zero eventually, which can be regarded as the limiting case of superconformal surfaces when the Hopf differential approaches zero. All minimal surfaces in $S^{3}$ or $S^{4}$ and all almost complex surfaces in $S^{6}$ are either superminimal or superconformal $([1],[14])$. Moreover, the existence of superconformal surfaces in higher dimensions is proved in [24].

In this paper, we determine in concrete terms the normalized potential of a superconformal surface in $S^{2 n}$ (the odd-dimensional case is included). In particular, when the Hopf differential is zero, the normalized potential embodies a first order system of equations governing superminimal surfaces in $S^{2 n}$ (see (8)), whose existence for any compact Riemann surfaces and arbitrarily large area can then be inferred accordingly.

After this paper had been completed we learned the recent appearance of Jun-Ichi Hano's paper ([17]), in which the same first order system for superminimal surfaces is derived and existence proven. So perhaps we should mention at the outset the salient part of our approach.

Hano's method rests on realizing the twistor space of $S^{2 n}$ through the $\operatorname{Spin}(2 n+1, \mathbb{C})$ representation and as a result the method is a delicate grouptheoretic analysis (see also [4] for a general but less explicit group-theoretic discussion). In comparison, our method is entirely algebro-geometric.

It was already implicit in Calabi's and explicit in Chern's work ([7],[8]) that a superminimal surface in $S^{2 n}$ is characterized by its directrix curve in $\mathbb{C} P^{2 n}$. Here, the directrix curve is the holomorphic curve generating the harmonic sequence of the surface, having the property that all the osculating $(n-1)$-planes of the directrix curve lie in the standard $(2 n-1)$-dimensional quadric $Q_{2 n-1}$ defined by

$$
x_{0}^{2}+x_{1}^{2}+\cdots+x_{2 n}^{2}=0
$$

$x_{j} \in \mathbb{C}$, in $\mathbb{C} P^{2 n}$. Hence the problem of determining superminimal surfaces for a fixed conformal structure is reduced to the algebro-geometric classification of those algebraic curves in the $(2 n-1)$-quadric with the property that all their osculating $(n-1)$-planes remain in the quadric. This naturally brings the twistor space $\mathcal{Z}_{2 n}$ of $S^{2 n}$ into the realm, which is the projective manifold consisting of all projective $(n-1)$-planes in the quadric $Q_{2 n-1}$, i.e., of all Euclidean $n$-planes in $\mathbb{C}^{2 n+1}$ satisfying (1) (called isotropic $n$-planes). Associated with the twistor space is the incidence space

$$
\mathcal{I}_{2 n}:=\left\{(p, V) \in Q_{2 n-1} \times \mathcal{Z}_{2 n}: p \in V\right\}
$$


which is a $\mathbb{C} P^{n-1}$-bundle over the twistor space.

Clearly, if a holomorphic curve in $Q_{2 n-1}$ has the property that all its osculating $(n-1)$-planes stay in $Q_{2 n-1}$, then the curve admits a holomorphic lift into $\mathcal{I}_{2 n}$ and into $\mathcal{Z}_{2 n}$. The latter is called the twistor lift of the curve.

We now outline a rather simple and somewhat novel method of deriving the first order system by looking only at $S^{6}$ for simplicity without any loss of generality. Let us be given the 5-quadric $Q_{5}$. By setting $w_{0}=x_{0}, z_{i}=$ $x_{i}+\sqrt{-1} x_{3+i}$ and $w_{i}=x_{i}-\sqrt{-1} x_{3+i}, 1 \leq i \leq 3,(1)$ is converted to a more convenient expression

$$
w_{0}^{2}+z_{1} w_{1}+z_{2} w_{2}+z_{3} w_{3}=0
$$

If an isotropic 3-plane $E$ is transversal to the $\left(w_{0}, w_{1}, w_{2}, w_{3}\right)$-plane, then $E$ is a graph over the $\left(z_{1}, z_{2}, z_{3}\right)$-plane, so that the $\left(w_{0}, w_{1}, w_{2}, w_{3}\right)$ coordinates of $E$ are linear functions of $z_{1}, z_{2}, z_{3}$, say,

$$
\begin{aligned}
& w_{0}=\alpha_{1} z_{1}+\alpha_{2} z_{2}+\alpha_{3} z_{3}, \\
& w_{1}=\sigma_{11} z_{1}+\sigma_{12} z_{2}+\sigma_{13} z_{3}, \\
& w_{2}=\sigma_{21} z_{1}+\sigma_{22} z_{2}+\sigma_{23} z_{3}, \\
& w_{3}=\sigma_{31} z_{1}+\sigma_{32} z_{2}+\sigma_{33} z_{3} .
\end{aligned}
$$

Inserting (3) through (6) into (2), we see that if we define

$$
\tau_{i j}=\sigma_{i j}+\alpha_{i} \alpha_{j}
$$

we have the identity

$$
\tau_{i j}=-\tau_{j i}
$$

Conversely, given any skew-symmetric $\tau_{i j}, 1 \leq i, j \leq 3$, and any triple $\alpha_{i}, 1 \leq i \leq 3$, we obtain an isotropic 3 -plane via the above formulae.

It is now clear that the collection of those isotropic 3-planes transversal to the $\left(w_{0}, \cdots, w_{3}\right)$-plane constitute an affine chart of $\mathcal{Z}_{6}$ parametrized by $\alpha_{1}, \alpha_{2}, \alpha_{3}, \tau_{12}, \tau_{23}, \tau_{31}$. Moreover, these coordinates together with $z_{1}, z_{2}, z_{3}$ form an affine chart of the incidence space $\mathcal{I}_{6}$.

Suppose we are given a curve in $Q_{5}$ such that all its osculating 2-planes stay in $Q_{5}$. Then the lift of the curve into $\mathcal{I}_{6}$, and hence the twistor lift into $\mathcal{Z}_{6}$, are described by (3) through (6), where $z_{1}, z_{2}, z_{3}$ are functions of a local uniformizing parameter $s$. Differentiating (3) through (6) with respect to $s$ twice, and keeping in mind the fact that the osculating 2planes all stay in $Q_{5}$, we see that we must have (3) through (6) valid as 
well when we replace $z_{1}, z_{2}, z_{3}$ and $w_{0}, w_{1}, w_{2}, w_{3}$ by their first and second derivatives. In particular, from (3) through (6) we arrive at the constraints, by differentiating once with respect to $s$,

$$
\begin{array}{r}
\alpha_{1}^{\prime} x+\alpha_{2}^{\prime} y+\alpha_{3}^{\prime} z=0, \\
\sigma_{11}^{\prime} x+\sigma_{12}^{\prime} y+\sigma_{13}^{\prime} z=0, \\
\sigma_{21}^{\prime} x+\sigma_{22}^{\prime} y+\sigma_{23}^{\prime} z=0, \\
\sigma_{31}^{\prime} x+\sigma_{32}^{\prime} y+\sigma_{33}^{\prime} z=0,
\end{array}
$$

for $(x, y, z)=\left(z_{1}, z_{2}, z_{3}\right)$ or $\left(z_{1}^{\prime}, z_{2}^{\prime}, z_{3}^{\prime}\right)$, which implies that the coefficient matrix of this homogeneous linear system is of rank one, i.e., any row is a multiple of another, or equivalently any 2-by-2 minors of the coefficient matrix is zero, which amounts to the equation

$$
d \sigma_{i j}=-2 \alpha_{i} d \alpha_{j}
$$

or equivalently,

$$
d \tau_{i j}=\alpha_{j} d \alpha_{i}-\alpha_{i} d \alpha_{j}
$$

These are precisely the equations for the twistor lift of a superminimal surface in $S^{2 n}$, which are also derived group-theoretically by Hano.

Bryant first wrote down (8) (a single equation) for $S^{4}$ in [2] through the quaternionic representation of $S^{4}$ as $\mathbb{H} P^{1}$ and its twistor space $\mathcal{Z}_{4}$ as the Hopf fibration over $\mathbb{H} P^{1}$, which is just $\mathbb{C} P^{3}$.

Bryant also studied superminimal almost complex curves in $S^{6}([3])$, which are governed by three equations different from those in (8) (see [3], or equation (40)). Of course, these equations must be consequences of (8) and almost complexity. After noting the beautiful property that the Frenet frame of a superminimal almost complex curve in $S^{6}$ is in fact a $G_{2}(\mathbb{C})$ frame, we derive in Section 3 that the twistor lift of an almost complex curve lies in a subvariety of $\mathcal{Z}_{6}$ birationally equivalent to the 5 -quadric given explicitly by the remarkably simple identity

$$
\tau_{23}=\sqrt{-1} \alpha_{1}
$$

(with an appropriate choice of the Frenet frame). From this there readily follow the equations for almost complex superminimal surfaces. Observe also that (9) implies that a generic superminimal surface in $S^{6}$ is not almost complex. We give a rigorous argument of this in Section 5 by exploiting the Riemann-Roch theorem. 
What is the degree of a superminimal surface in $S^{2 n}$ ? In Section 4, we show that the degree of a compact superminimal surface in $S^{6}$, which is the degree of its holomorphic lift in the twistor space $\mathcal{Z}_{6}$, is half the degree of the projective curve

$$
\left[1: \alpha_{1}: \alpha_{2}: \alpha_{3}: \tau_{12}: \tau_{23}: \tau_{31}\right]
$$

in $\mathbb{C} P^{6}$ as long as the surface does not pass through the north pole. Our method is a straightforward calculation based on the Plücker embedding of the twistor space $\mathcal{Z}_{6}$ into $\mathbb{C} P^{34}$. However, such a method will clearly get rather complicated as the dimension of the sphere gets large. In this regard we mention that the same fact about the degree of a superminimal surface remains true in an appropriate generic sense for any $S^{2 n}$, which is proven in [15] using different arguments. An important implication of this result is that the moduli space of superminimal surfaces in $S^{2 n}$ of a fixed degree with a given conformal structure in $S^{2 n}$ is entirely characterized by the above corresponding curves in $\mathbb{C} P^{n(n+1) / 2}$, so that the study of such moduli spaces can now be carried out in the projective space ([15]) instead of the more complicated twistor space, which is only birationally equivalent to $\mathbb{C} P^{n(n+1) / 2}$. Note that this degree property is obviously true for $S^{4}$ since $\mathcal{Z}_{4}$ is isomorphic to $\mathbb{C} P^{3}$; see [10] for the related moduli space problem.

Another consequence of this degree property is that there exist superminimal surfaces of arbitrarily large degree (or area) in $S^{2 n}$. A proof of this fact for $S^{6}$ is given in Section 5 , where we prove the existence of superminimal surfaces, not almost complex, of any genus and arbitrarily large degree. The general case is similar.

We conclude the paper by determining in Section 6 the normalized potential of a superconformal surface in $S^{2 n}$ in terms of certain normal curvatures of the surface, and relate it to the superminimal case by letting the Hopf differential be zero. This way we gain a deeper geometric insight into the recursive formulae defining the normalized potential in the superminimal case obtained in Section 1 through different means.

\section{$\S 1$. Weierstrass-type representation and the normalized potential}

1.1. We first review the Weierstrass-type representation for primitive harmonic maps from a Riemann surface $M$ to a compact $k$-symmetric space and its associated normalized potential developed in ([12]).

Let $G / K$ be a compact Riemannian $k$-symmetric space with $k$-involution $\sigma: G \longrightarrow G$ so that $G_{0}^{\sigma} \subset K \subset G^{\sigma}$, where $G_{0}^{\sigma}$ is the connected 
component containing the identity in the fixed point group $G^{\sigma}$ of $\sigma$. We denote by $\mathcal{G}$ and $\mathcal{K}$ the Lie algebra of $G$ and $K$, respectively, and embed $G$ into a $G L(m, \mathbb{R})$ for some $m$ once and for all. The decomposition $\mathcal{G}^{\mathbb{C}}$ into $\omega^{l}$-eigenspaces, where $\omega$ is a $k$-th root of unity, of the automorphism $\sigma: \mathcal{G}^{\mathbb{C}} \longrightarrow \mathcal{G}^{\mathbb{C}}$ gives the Cartan-like decomposition

$$
\mathcal{G}^{\mathbb{C}}=\bigoplus_{l=0}^{k-1} \mathcal{G}_{l}^{\mathbb{C}}
$$

with $\mathcal{G}_{0}^{\mathbb{C}}=\mathcal{K}^{\mathbb{C}}$. The indices $l$ in $\mathcal{G}_{l}^{\mathbb{C}}$ are understood to be integers $\bmod k$. Let $\tau: G \longrightarrow G / K$ be the coset projection, $M$ a Riemann surface and $f: M \longrightarrow G / K$ a smooth map. Let $F: M \longrightarrow G$ be a frame of $f$, i.e., $\tau \circ F=f$. We say $f$ is primitive $([5])$ if $f$ has a frame $F$ such that

$$
\partial F \cdot F^{-1} \in \mathcal{G}_{0}^{\mathbb{C}} \oplus \mathcal{G}_{1}^{\mathbb{C}} .
$$

In fact, it is easy to see that if $f$ is primitive, then all frames of $f$ satisfy (11). Let us introduce the loop groups

$$
\begin{aligned}
& \bigwedge G_{\sigma}^{\mathbb{C}}=\left\{g: S^{1} \longrightarrow G^{\mathbb{C}} ; g(\omega \lambda)=\sigma g(\lambda) \text { for all } \lambda \in S^{1}\right\}, \\
& \bigwedge G_{\sigma}=\left\{g \in \bigwedge G_{\sigma}^{\mathbb{C}}: g(\lambda) \in G \text { for all } \lambda \in S^{1}\right\} .
\end{aligned}
$$

When given an $H^{s}$-topology with $s>1 / 2, \bigwedge G_{\sigma}^{\mathbb{C}}$ is a complex Banach Lie group. Then one has the following $([12])$.

Proposition 1. Let $M$ be a contractible simply connected Riemann surface. Then $f: M \longrightarrow G / K$ is primitive harmonic if and only if there exists a map $\Phi: M \longrightarrow \bigwedge G_{\sigma}, \Phi: p \longmapsto \Phi(p, \lambda)$, satisfying $\tau \circ \Phi(\cdot, 1)=f$ and

$$
d \Phi \cdot \Phi^{-1}=\overline{\alpha_{1}} \lambda^{-1}+\alpha_{0}+\alpha_{1} \lambda
$$

for some 1 -form $\alpha_{0}: T M \longrightarrow \mathcal{G}_{0}=\mathcal{K}$ and some $(1,0)$-form $\alpha_{1}: T M \longrightarrow$ $\mathcal{G}_{1}^{\mathbb{C}}$.

Since $M$ is simply connected, we can assume that $M$ is a region in $\mathbb{C}$ containing the origin. The map $\Phi$ in the proposition is called an extended frame of $f$ if it satisfies the initial condition

$$
\Phi(0, \cdot)=F(0)
$$

where $F$ is the frame of $f$ used to derive $\alpha_{0}$ and $\alpha_{1}$. Therefore the problem of constructing the primitive harmonic maps from a simply connected $M$ 
to $G / K$ becomes a problem of constructing the extended frames from $M$ to $\bigwedge G_{\sigma}$.

Before proceeding, let us introduce three more loop groups. Let $K^{\mathbb{C}}=$ $K \cdot B$ be an Iwasawa decomposition, where $B \subset K^{\mathbb{C}}$ is a solvable subgroup such that $K \cap B=\{I\}$, where $I$ the identity element. We set

$\bigwedge^{-} G_{\sigma}^{\mathbb{C}}=\left\{g \in \bigwedge_{\sigma}^{\mathbb{C}}: g\right.$ extends holomorphically to $\left.|z|>1\right\}$,

$\bigwedge_{*}^{+} G_{\sigma}^{\mathbb{C}}=\left\{g \in \bigwedge G_{\sigma}^{\mathbb{C}}: g\right.$ extends holomorphically to $|z|<1$ and $\left.g(0)=I\right\}$, $\bigwedge_{B}^{-} G_{\sigma}^{\mathbb{C}}=\left\{g \in \bigwedge^{-} G_{\sigma}^{\mathbb{C}}: g(\infty) \in B\right\}$.

The multiplication

$$
\bigwedge_{B}^{-} G_{\sigma}^{\mathbb{C}} \times \bigwedge G_{\sigma} \longrightarrow \bigwedge G_{\sigma}^{\mathbb{C}}
$$

is an onto diffeomorphism, and the multiplication

$$
\bigwedge^{-} G_{\sigma}^{\mathbb{C}} \times \bigwedge_{*}^{+} G_{\sigma}^{\mathbb{C}} \longrightarrow \bigwedge_{\sigma}^{\mathbb{C}}
$$

is a diffeomorphism onto the open and dense subset $\bigwedge^{-} G_{\sigma}^{\mathbb{C}} \cdot \bigwedge_{*}^{+} G_{\sigma}^{\mathbb{C}}([12])$. Then the following generalization of Lemma 4.5 and Theorem 4.10 in ([12]) gives a recipe for the construction of such primitive harmonic maps.

THEOREM 1. (i) Let $M$ be simply connected and parametrized by $z$ around 0 , and let $f: M \longrightarrow G / K$ be primitive harmonic with $f(0)=I K$ and $\Phi: M \longrightarrow \bigwedge G_{\sigma}$ an extended frame of $f$. Then there is a discrete set $S \subset M \backslash\{0\}$ such that for $z \in M \backslash S$,

$$
\Phi(z, \cdot)=\Phi_{-}(z, \cdot) \Phi_{+}(z, \cdot)
$$

with $\Phi_{-}(z, \cdot) \in \bigwedge^{-} G_{\sigma}^{\mathbb{C}}$ and $\Phi_{+}(z, \cdot) \in \bigwedge_{*}^{+} G_{\sigma}^{\mathbb{C}}$, and

$$
P(z)=d \Phi_{+}(z, \lambda) \cdot \Phi_{+}(z, \lambda)^{-1} \cdot \lambda^{-1}
$$

is a $\mathcal{G}_{+1}^{\mathbb{C}}$-valued meromorphic $(1,0)$-form with poles in $S$.

(ii) Conversely, any primitive harmonic map with $f(0)=I K$ can be constructed from a $\mathcal{G}_{+1}^{\mathbb{C}}$-valued meromorphic 1 -form $P$ on $M$ by integrating

$$
d \Phi_{+}(z, \lambda)=P(z) \Phi_{+}(z, \lambda) \cdot \lambda, \quad \Phi_{+}(0, \cdot)=I
$$

to get $\Phi_{+}$and then obtaining an extended frame $\Phi$ via the factorization

$$
\Phi_{+}(z, \cdot)=\Phi_{-}(z, \cdot)^{-1} \Phi(z, \cdot)
$$

given in (13). 
The (1,0)-form $P$ in the theorem, unique up to the conjugations by the elements of $K$, is called the normalized potential for $f$ with the origin as the reference point. The construction from $P$ to a frame $\Phi$ may be thought of as a kind of Weierstrass-type representation for primitive harmonic maps.

1.2. Theorem 1 is quite explicit in the case of a holomorphic curve in $\mathbb{C} P^{n}$. The complex flag manifold $U(n+1) / U(1) \times \cdots \times U(1)$ is an $(n+1)$-symmetric space with the involution $\sigma$ equal to the conjugation by $\operatorname{diag}\left(\omega^{n}, \cdots, \omega, 1\right)$, where $\omega$ is an $(n+1)$-th root of unity. Let

$$
\left[a_{00}=1: a_{01}: \cdots: a_{0 n}\right]
$$

be a linearly full holomorphic curve, where the coordinates are meromorphic functions on a Riemann surface $M$. A frame $F$ of the curve is just a Frenet frame, i.e., is a unitary frame obtained by applying the GramSchmidt process to the osculating curves of the curve, i.e., to the derivatives $f, f^{\prime}, f^{\prime \prime}, \cdots, f^{(n)}$ of a local lift $f$ of the curve into $\mathbb{C}^{n+1}$. Then it is directly checked that for $\lambda \in S^{1}$, the frame

$$
F_{\lambda}=\Lambda F \Lambda^{-1}
$$

with $\Lambda=\operatorname{diag}\left(\lambda^{n}, \cdots, \lambda, 1\right)$ solves $(12)$, so that $F_{\lambda}$ is an extended frame if we assume without loss of generality that $\mathrm{F}(0)=\mathrm{I}$. The decomposition

$$
F_{\lambda}=\left(F_{\lambda}\right)_{-}\left(F_{\lambda}\right)_{+}
$$

in (15) is achieved by the row reduction process applied to $F_{\lambda}$, so that $F_{\lambda}$ is the multiplication of a lower triangular matrix $\left(F_{\lambda}\right)_{-}$and an upper triangular matrix $\left(F_{\lambda}\right)_{+}$, which are polynomials in $\lambda^{-1}$ and $\lambda$, respectively. To find $\left(F_{\lambda}\right)_{+}$, we observe that it is equivalent to upper-triangularizing the Wronskian matrix of the row vector in (19) such that the resulted diagonal entries are all 1 . We then conclude that the first row of $\left(F_{\lambda}\right)_{+}$is the vector

$$
\left(a_{00}, a_{01} \lambda, a_{02} \lambda^{2}, \cdots, a_{0 n} \lambda^{n}\right)
$$

and in general the $(k+1)$-th row is obtained by differentiating the $k$-th row and dividing by the $(k+1)$-th coordinate of the resulting differentiated row. This leads to the recursive formulae defining $\left(F_{\lambda}\right)_{+}$, i.e.,

$$
\left(F_{\lambda}\right)_{+}=\left(a_{i j} \lambda^{j-i}\right)
$$


where

$$
a_{i j}=\frac{d a_{i-1, j}}{d a_{i-1, i}}
$$

if $1 \leq i<j$, and 1 on the diagonal and 0 elsewhere.

As a consequence, the meromorphic potential $P$ is the matrix 1-form which is 0 everywhere except for the $(i, i+1)$-th entries given by

$$
d a_{i, i+1}
$$

for $0 \leq i \leq n-1$.

Conversely, when $P$ is given, an iterated integration via (21) and (22) derives the curve (19).

Remark 1. That equation (20) satisfies equation (12) is a consequence of the rigidity of holomorphic curves in complex projective spaces $([6],[9])$. Equation (20) means that we have a 1-parameter family of isometric holomorphic curves which must at the same time be unitarily equivalent because of the rigidity property.

Definition 1. By a superminimal surface of location $k$ in $\mathbb{C} P^{n}$ we mean a surface which is the projectivization of the $k$-th vector in a Frenet frame of a holomorphic curve, called the directrix curve of the surface.

Let $U$ be the matrix obtained by interchanging the first $k$ rows and the remaining $(n-k+1)$ rows of the $(n+1) \times(n+1)$ identity matrix. The following proposition is then easy to see in view of (16).

Proposition 2. The normalized potential of a linearly full superminimal surface of location $k$ in $\mathbb{C} P^{n}$ is the matrix 1 -form $U P U^{-1}$ with $P$ given by $(22)$.

Definition 2. By a superminimal surface in $S^{n}$ we mean a surface which is superminimal when it is regarded as sitting in $\mathbb{C} P^{n}$ via the standard immersion $S^{n} \longrightarrow \mathbb{C} P^{n}$.

Let $f_{0}$ be the position vector of a superminimal surface in $\mathbb{R}^{n+1}$ and let

$$
\left(f_{-k}, \cdots, f_{0}, \cdots f_{l}\right), \quad k, l \geq 1
$$

be a Frenet frame of the directrix curve $\left[f_{-k}\right]$ of the surface $f_{0}$. The following proposition is well-known $([8])$. 
Proposition 3. (i) Notation as above, we have $k=l, f_{-s}=\overline{f_{s}}$ for $s \neq 0$ and $\left(f_{s}, f_{t}\right)=0$ for all $s, t<0$, where $($,$) denotes the symmetric$ product of $\mathbb{C}^{n+1}$. Hence all the (projective) osculating $(k-1)$-planes of the directrix curve live in the standard $(n-1)$-quadric

$$
z_{0}^{2}+\cdots+z_{n}^{2}=0
$$

in $\mathbb{C} P^{n}$; in particular, only even-dimensional spheres can contain a linearly full superminimal surface.

(ii) Conversely, a linearly full holomorphic curve in the standard (2n$1)$-quadric in $\mathbb{C} P^{2 n}$ whose osculating $(n-1)$-planes are all contained in the quadric gives rise to a linearly full superminimal surface in $S^{2 n}$.

In accordance with the preceding proposition, we will assume linear fullness of superminimal surfaces in $S^{2 n}$ from now on.

The normalized potential of the directrix curve of a superminimal surface in $S^{2 n}$ is given by (22) together with $n$ more linear constraints.

Proposition 4. The normalized potential of the directrix curve of a superminimal surface in $S^{2 n}$ is the matrix form $P$ given by $(22)$ such that

$$
\begin{aligned}
& d a_{01}=-d a_{2 n-1,2 n}, \\
& d a_{12}=-d a_{2 n-2,2 n-1}, \\
& \vdots \\
& d a_{n-2, n-1}=-d a_{n+1, n+2}, \\
& 2 d a_{n-1, n}=-d a_{n, n+1} .
\end{aligned}
$$

Proof. Let $e_{0}, \cdots, e_{2 n}$ be the standard basis of $\mathbb{C}^{2 n+1}$ and let $E_{0}=$ $e_{0}, E_{ \pm 1}=\left(e_{1} \mp \sqrt{-1} e_{2}\right) / 2, E_{ \pm 2}=\left(e_{3} \mp \sqrt{-1} e_{4}\right) / 2, \cdots, E_{ \pm n}=\left(e_{2 n-1} \mp\right.$ $\left.\sqrt{-1} e_{2 n}\right) / 2$. A straightforward calculation shows that when a matrix in $s o(2 n+1, \mathbb{R})$ is written as a matrix $\left(p_{i j}\right)$ relative to the basis $E_{-n}, \cdots, E_{0}, \cdots$, $E_{n}$ with the property that all entries, except for the ones on the main diagonal and on the two off-diagonals, are zero, then we have $p_{01}=-p_{2 n-1,2 n}, p_{12}$ $=-p_{2 n-2,2 n-1}, \cdots, p_{n-2, n-1}=-p_{n+1, n+2}, 2 p_{n-1, n}=-p_{n, n+1}$.

We remark that in the above proposition, the factor 2 , instead of $\sqrt{2}$, in the definition of the basis elements $E_{i}$ is chosen to avoid the factor $1 / 2$ that would otherwise appear in front of $\left(a_{i n}\right)^{2}$ in $(23)$ below. It is clear that 
the difference in the choice only results in a rescaling of the coordinates of the directrix curve.

1.3. Of course, the formulae in Proposition 4, although in the disguise of complicated "towers" of $d$, are in fact equivalent to saying that the osculating $(n-1)$-planes of the directrix curve lie entirely in the quadric in view of Theorem 1 and Proposition 3. We will next indicate why this is true by "peeling off $d$ " from the formulae in Proposition 4 by continuing to integrate by parts.

Proposition 5. Let $\left(a_{i j}\right)$ be the upper triangular matrix given by $(21)$, $0 \leq i<j \leq 2 n$, with diagonal entries 1 . If $\left(a_{i j}\right)$ satisfies the condition in Proposition 4, then

$$
\left(a_{i n}\right)^{2}+\sum_{j=0}^{n-1} a_{i j} a_{i, 2 n-j}=0
$$

for $0 \leq i \leq n-1$

Proof. We will indicate a proof for $S^{6}$ for simplicity. The general case is similar. For $n=3$, the equations of Proposition 4 are

$$
\begin{gathered}
2 a_{23}=-a_{34}, \\
a_{12}=-a_{45}, \\
a_{01}=-a_{56} .
\end{gathered}
$$

(We may ignore integrating constants because they amount to only creating linear changes of coordinates.) Now (21) gives $a_{34}=d a_{24} / d a_{23}$ so that we have, by (24), $2 a_{23} d a_{23}=-d a_{24}$. Integrating yields

$$
\left(a_{23}\right)^{2}+a_{24}=0
$$

which is $(23)$ for $i=2$.

From (21) and (25) we derive $d a_{35}=2 d\left(a_{12} a_{23}-a_{13}\right)$ so that (21) gives

$$
d a_{25}=2\left(a_{12} a_{23}-a_{13}\right) d a_{23}
$$

Incorporating (27) and (21) again and integrating by parts yields

$$
a_{25}=-a_{12} a_{24}-a_{14}-2 a_{13} a_{23}
$$


which is equivalent to

$$
d a_{15}=d\left(-a_{12} a_{14}-a_{13}^{2}\right)
$$

which is (23) for $i=1$.

Equation (26) is equivalent to

$$
d a_{46}=d\left(a_{01} a_{12}-a_{02}\right)
$$

by (25), so that we integrate and employ (21) to give

$$
d a_{36}=-2 d\left(\left(a_{01} a_{12}-a_{02}\right) a_{23}-a_{01} a_{13}+a_{03}\right) .
$$

Repeating the same process results in

(30) $d a_{26}=d\left(\left(a_{01} a_{12}-a_{02}\right) a_{24}+a_{01} a_{14}-a_{04}-2\left(-a_{01} a_{13}+a_{03}\right) a_{23}\right)$,

and consequently by integrating we obtain

$$
d a_{16}=-d\left(a_{05}+a_{01} a_{15}+a_{04} a_{12}+a_{02} a_{14}+2 a_{03} a_{13}\right) .
$$

Integrating (31) and employing $a_{16}=d a_{06} / d a_{01}$ finally arrives at (23) for $i=0$.

A consequence of Proposition 5 is that, when comparing (23) with (3) through (6), we see that for each fixed $i, 0 \leq i \leq n-1$, the array $a_{i, k+n}, 0 \leq$ $k \leq n$, in (23), plays the role of $w_{k}, 0 \leq k \leq n$, in (3) through (6); likewise for each fixed $i$ the array $a_{i j}, 0 \leq j \leq n-1$, in (23) corresponds to $z_{j}, 1 \leq j \leq n$ in (3) through (6). Hence when varying $i$ we may, in accordance with the matrix setup of (3) through (6), conveniently phrase (23) in the following matrix equation

$$
\left(a_{i, k+n}\right)=\left(a_{i j}\right) B
$$

where $0 \leq i, j \leq n-1,0 \leq k \leq n$, and $B$ is the $n \times(n+1)$ matrix such that the first column of which is $\left(\alpha_{1}, \cdots, \alpha_{n}\right)^{t r}$ and the $i$-th column, $2 \leq i \leq n+1$, of which is $\left(\sigma_{i-1,1}, \cdots, \sigma_{i-1, n}\right)^{t r}$. In other words, from the solution of $(23)$, the matrix

$$
\left(a_{i j}\right)^{-1}\left(a_{j, k+n}\right)
$$

$0 \leq i, j \leq n-1,0 \leq k \leq n$, contains precisely $\alpha_{i}$ and $\sigma_{i j}$ which satisfy

$$
d \sigma_{i j}=-2 \alpha_{i} d \alpha_{j}
$$

for $1 \leq i, j \leq n$. This is explicitly how the normalized potential determines the twistor lift of the superminimal surface. 


\section{$\S 2 . \quad$ More quadric geometry}

We have seen in the introduction that (3) through (6) remain true when we replace $z_{1}, z_{2}, z_{3}$ and $w_{0}, w_{1}, w_{2}, w_{3}$ respectively by their 1 st and 2 nd derivatives; in fact, (7) is a consequence of substituting the 1st derivatives. When we replace $z_{1}, z_{2}, z_{3}$ by their 2 nd derivatives in (3), we derive the constraints

$$
\begin{aligned}
& \alpha_{1}^{\prime} z_{1}+\alpha_{2}^{\prime} z_{2}+\alpha_{3}^{\prime} z_{3}=0 \\
& \alpha_{1}^{\prime \prime} z_{1}+\alpha_{2}^{\prime \prime} z_{2}+\alpha_{3}^{\prime \prime} z_{3}=0 .
\end{aligned}
$$

In view of (23) and (3) through (6), we may assume $a_{00}=z_{1}=1, a_{01}=z_{2}$, and $a_{02}=z_{3}$. Setting $z_{1}=1$ and solving for $z_{2}$ and $z_{3}$ in terms of $\alpha_{1}, \alpha_{2}, \alpha_{3}$ establishes

$$
\begin{aligned}
& a_{01}=-\frac{d^{2} \alpha_{1}}{d \alpha_{3}^{2}} / \frac{d^{2} \alpha_{2}}{d \alpha_{3}^{2}} \\
& a_{02}=-\frac{d^{2} \alpha_{1}}{d \alpha_{2}^{2}} / \frac{d^{2} \alpha_{3}}{d \alpha_{2}{ }^{2}}
\end{aligned}
$$

The point is that, conversely, we can express the holomorphic curve

$$
\left[a_{00}=1: a_{01}: \cdots: a_{06}\right]
$$

and hence its normalized potential (22), in terms of $\alpha_{i}, 1 \leq i \leq 3$ and $\sigma_{i j}, 1 \leq i \neq j \leq 3$, as soon as they satisfy (7). Indeed, by the definition of $\alpha_{i}$ and $\sigma_{i j}, 1 \leq i \neq j \leq 3$, in (3) through (6), we see

$$
\begin{aligned}
& a_{03}=\sum_{i=0}^{2} \alpha_{i+1} a_{0 i}, \\
& a_{0 k}=\sum_{i=1}^{3} \sigma_{7-k, i} a_{0 i},
\end{aligned}
$$

for $4 \leq k \leq 6$. We leave it to the reader to verify that the curve determined in this way has the property that all the osculating 2-planes of the curve live in the 5-quadric.

It is clear that, although we carried it out only for $S^{6}$ for later purposes, a similar statement is true for all $S^{2 n}$. 


\section{$\S 3$. Almost complex superminimal surfaces in $S^{6}$}

Recall that the Cayley algebra $\mathbb{O}$ is defined by $\mathbb{H} \oplus \mathbb{H}$ with the multiplication

$$
(a, b) \cdot(c, d)=(a c-\bar{d} b, d a+b \bar{c}) .
$$

Writing $(0,1)$ as $\varepsilon$ we see $\mathbb{O}$ is generated by $(1, i, j, k, \varepsilon, i \varepsilon, j \varepsilon, k \epsilon)$. For $x, y \in$ $\operatorname{Im} \mathbb{O}$, the imaginary part of $\mathbb{O}$ generated by $i, j, \cdots, k \varepsilon$, we can define the cross product

$$
x \times y=(x \cdot y-y \cdot x) / 2 .
$$

Now identify $S^{6}$ with the unit sphere in $\operatorname{Im} \mathbb{O}$ with the usual inner product equal to

$$
<x, y>=-(x \cdot y+y \cdot x) / 2 .
$$

Then $S^{6}$ carries the almost complex structure

$$
J_{u}(x)=u \times x
$$

for $u \in S^{6}$ and $x \perp u$ regarded as a vector in $T_{u} S^{6}$.

$J$ splits $T_{u}^{\mathbb{C}} S^{6}$ into the (1,0)-part and the $(0,1)$-part, which are the eigenspaces of $J$ with eigenvalues $\sqrt{-1}$ and $-\sqrt{-1}$, respectively.

A smooth map $f: M \longrightarrow S^{6}$ from a Riemann surface into $S^{6}$ is said to be an almost complex surface if $d f$ preserves the almost complex structure, i.e., if $z$ is a local uniformizing parameter of $M$, then

$$
f \times f_{z}=\sqrt{-1} f_{z},
$$

where as usual $f_{z}$ denotes partial differentiation with respect to $z$, etc. Differentiating (36) we get

$$
f \times f_{z z}=\sqrt{-1} f_{z z} .
$$

Now let $z=x+\sqrt{-1} y$. Then the real version of (36) is $f \times f_{x}=f_{y}$ and $f \times f_{y}=-f_{x}$. In particular $f \times f_{z \bar{z}}=0$. As a result $f_{z \bar{z}}$ is parallel to $f$, which implies that an almost complex surface is automatically a branched minimal immersion into $S^{6}$ with the induced metric.

When $f$ is a superminimal almost complex surface, on the one hand we have, from Proposition 3 , that the Frenet frame $\left(f_{-3}, f_{-2}, f_{-1}, f_{0}=\right.$ $\left.f, f_{1}, f_{2}, f_{3}\right)$ with $f_{-k}=\overline{f_{k}}$ and $f_{k}$ are quadric vectors for all nonzero $k$. On the other hand,

$$
f_{3}=f_{1} \times f_{2}
$$


up to a constant multiple. To see this, we observe that since $f_{1}$ and $f_{2}$ are $(1,0)$-vectors of $J$ by $(36)$ and $(37)$, we can pick a local orthonormal frame $e_{1}, \cdots, e_{7}$ such that

$$
e_{3}=e_{1} \times e_{2}, e_{5}=e_{1} \times e_{4}, e_{6}=e_{2} \times e_{4}, e_{7}=e_{3} \times e_{4}
$$

with

$$
f=e_{4}, f_{1}=\left(e_{1}-\sqrt{-1} e_{5}\right) / 2, f_{2}=\left(e_{2}-\sqrt{-1} e_{6}\right) / 2 .
$$

It is then easy to see that

$$
f_{1} \times f_{2}=\left(e_{3}+\sqrt{-1} e_{7}\right) / 2 .
$$

Moreover, $f_{3}$ is spanned by

$$
E:=\left(e_{3}-\sqrt{-1} e_{7}\right) / 2
$$

and $\bar{E}\left(=f_{1} \times f_{2}\right)$. Set

$$
f_{3}=a E+b \bar{E}
$$

for some functions $a$ and $b$.

Let us write out the Frenet frame:

$$
\begin{aligned}
d f & =A f_{1} d z+A^{\prime} f_{-1} d \bar{z} \\
d f_{1} & =B f_{2} d z+B^{\prime} f d \bar{z} \bmod \left(f_{1}\right), \\
d f_{2} & =C f_{3} d z+C^{\prime} f_{1} d \bar{z} \bmod \left(f_{2}\right) \\
d f_{3} & =D^{\prime} f_{2} d \bar{z} \bmod \left(f_{3}\right)
\end{aligned}
$$

for some nonzero functions $A, B, C, A^{\prime}, B^{\prime}, C^{\prime}, D^{\prime}$ with $f_{-3}$ the directrix curve of $f$. Differentiating and employing the Frenet frame we get

$$
\begin{aligned}
\bar{E}_{z} & =C f_{1} \times f_{3} \bmod (\bar{E}) \\
& =-a C f_{-2} \bmod (\bar{E}) .
\end{aligned}
$$

It follows that if $a \neq 0, \bar{E}=f_{1} \times f_{2}$ will be the directrix curve of $f$, so that

$$
\bar{E}_{\bar{z}}=0 \bmod (\bar{E}) \text {. }
$$

However, a calculation using the Frenet frame shows that

$$
\begin{aligned}
\bar{E}_{\bar{z}} & =B^{\prime} f \times f_{2} \bmod (\bar{E}) \\
& =\sqrt{-1} B^{\prime} f_{2} \bmod (\bar{E}) \\
& \neq 0 \bmod (\bar{E}) .
\end{aligned}
$$


This contradiction establishes that $a=0$ in (39). Hence (38) is true; in particular, we have the beautiful property that the Frenet frame of an almost complex superminimal surface is in fact a $G_{2}(\mathbb{C})$-frame.

The directrix curve of an almost complex superminimal surface now has the feature that its osculating 2-planes are spanned by one $(1,0)$-vector and two $(0,1)$-vectors. We wish to characterize these osculating planes in the twistor space $\mathcal{Z}$. To this end we employ (38) to see that in the Frenet frame above we have

$$
C=\sqrt{-1} A / 2,
$$

or equivalently in the notations of Sections 1 and 2

$$
\begin{aligned}
a_{01} & =\sqrt{-1} a_{23} \\
& =\sqrt{-1} \alpha_{3} .
\end{aligned}
$$

In light of (33) we have consequently the differential equation

$$
-\sqrt{-1} \alpha_{3} \frac{d}{d \alpha_{3}}\left(\frac{d \alpha_{2}}{d \alpha_{3}}\right)=\frac{d}{d \alpha_{3}}\left(\frac{d \alpha_{1}}{d \alpha_{3}}\right)
$$

Integrating by parts, we get

$$
-\frac{d \alpha_{1}}{d \alpha_{3}}=\sqrt{-1} \alpha_{3} \frac{d \alpha_{2}}{d \alpha_{3}}-\sqrt{-1} \alpha_{2}
$$

which amounts to the same as

$$
-d \alpha_{1}=\sqrt{-1}\left(\alpha_{3} d \alpha_{2}-\alpha_{2} d \alpha_{3}\right) .
$$

In other words, we have obtained the remarkably simple identity

$$
\tau_{23}=\sqrt{-1} \alpha_{1}
$$

We have thus shown the following.

Proposition 6. The directrix curve of an almost complex superminimal surface in $S^{6}$ is described in a suitable coordinate by (8) with the linear constraint $\tau_{23}=\sqrt{-1} \alpha_{1}$, and vice versa. 
Let us now introduce the new variables

$$
\begin{aligned}
y & :=\tau_{23}\left(=\sqrt{-1} \alpha_{1}\right), \\
x_{2} & :=-\sqrt{-1} \alpha_{2}, \\
x_{3} & :=-\sqrt{-1} \alpha_{3}, \\
z_{2} & :=\sigma_{21}+\frac{\sqrt{-1}}{2}\left(\alpha_{2}\right)^{2} \alpha_{3}, \\
z_{3} & :=\sigma_{31}-\frac{\sqrt{-1}}{2} \alpha_{2}\left(\alpha_{3}\right)^{2} .
\end{aligned}
$$

Then it is readily verified by (7) or (8) that we have

$$
\begin{aligned}
d y & =x_{2} d x_{3}-x_{3} d x_{2} \\
d z_{2} & =-3 x_{2} d y+\frac{3}{2}\left(x_{2}\right)^{2} d x_{3} \\
d z_{3} & =-3 x_{3} d y-\frac{3}{2}\left(x_{3}\right)^{2} d x_{2}
\end{aligned}
$$

These are exactly the equations that Bryant derived in $([3])$, in a more complicated fashion, for almost complex superminimal surfaces.

\section{$\S 4$. Degree of a superminimal surface}

The area of a compact superminimal surface in $S^{2 n}$ is a constant multiple of the degree of the $(n-1)$-th associated curve, i.e., the curve of osculating $(n-1)$-planes, of its directrix curve when the associated curve is regarded as sitting in $\mathbb{P}\left(\wedge^{n}\left(\mathbb{C}^{2 n+1}\right)\right)$ via the Plücker embedding. We refer to this degree as the degree of the superminimal surface. Although $\mathcal{Z}$ is in general not a projective space, we will show in this section that the degree of a compact superminimal surface in $S^{6}$ not passing through the north pole is completely determined by a certain projective curve in $\mathbb{C} P^{6}$. More precisely, we have the following.

THEOREM 2. Let the projective curve $\left[1: \alpha_{1}: \alpha_{2}: \alpha_{3}: \tau_{12}: \tau_{23}: \tau_{31}\right]$ in $\mathbb{C} P^{6}$, where the coordinates satisfy (8), be such that the corresponding superminimal surface does not pass through the north pole. Then the curve has degree $d$ if and only if the 2nd associated curve in $\mathcal{Z}_{6}$ of the corresponding directrix curve defined by (3) through (6) has degree $2 d$ in $\mathbb{C} P^{34}$.

An important implication of this theorem is that the moduli space of superminimal surfaces of a fixed degree in $S^{6}$ is now entirely characterized 
by the moduli space of the projective curves of a fixed degree in $\mathbb{C} P^{6}$ given in the theorem.

Theorem 2 has been generalized to $S^{2 n}$ in ([15]) by different arguments. As a consequence, the moduli space of superminimal surfaces of a fixed degree in $S^{2 n}$ has been set up and its connectedness has been proven in an explicit geometric way, in contrast with the earlier proof of connectedness in ([21]) for the case of genus zero, and ([16]) for arbitrary genus via loop-group analysis.

To prove Theorem 2, let $f: M \longrightarrow \mathbb{C} P^{6}$ be the curve $\left[1: \alpha_{1}: \alpha_{2}:\right.$ $\left.\alpha_{3}: \tau_{12}: \tau_{23}: \tau_{31}\right]$. Then the coordinates of $f$ may be regarded as sections $s_{0}, \cdots, s_{6}$ of $f^{-1} \mathcal{O}(\infty)$, without common zeros, such that

$$
\alpha_{1}=s_{1} / s_{0}, \alpha_{2}=s_{2} / s_{0}, \cdots, \tau_{31}=s_{6} / s_{0}
$$

In view of $(3)$ through $(6)$, by setting $\left(z_{1}, z_{2}, z_{3}\right)$ equal to $(1,0,0),(0,1,0)$ and $(0,0,1)$ respectively we have three quadric vectors

$$
\begin{aligned}
& W_{1}=\left(\alpha_{1}, 1,0,0, \sigma_{11}, \sigma_{21}, \sigma_{31}\right), \\
& W_{2}=\left(\alpha_{2}, 0,1,0, \sigma_{12}, \sigma_{22}, \sigma_{32}\right), \\
& W_{3}=\left(\alpha_{3}, 0,0,1, \sigma_{13}, \sigma_{23}, \sigma_{33}\right),
\end{aligned}
$$

which generate the osculating 2-planes of the corresponding directrix curve. The projectivization of $W_{1} \wedge W_{2} \wedge W_{3}$ embeds these osculating 2-planes into $\mathbb{C} P^{34}$. Let $e_{1}, \cdots, e_{7}$ be the standard basis of $\mathbb{C}^{7}$. We will only display the coordinates of $W_{1} \wedge W_{2} \wedge W_{3}$ that are crucial to the proof:

$$
\begin{aligned}
& \quad e_{2} \wedge e_{3} \wedge e_{4} \\
& +\alpha_{3} e_{1} \wedge e_{2} \wedge e_{3} \\
& +\cdots \\
& -\alpha_{1}^{2} e_{3} \wedge e_{4} \wedge e_{5}+\alpha_{2}^{2} e_{2} \wedge e_{4} \wedge e_{6}-\alpha_{3}^{2} e_{2} \wedge e_{3} \wedge e_{7} \\
& +\tau_{12}^{2} e_{4} \wedge e_{5} \wedge e_{6}+\tau_{23}^{2} e_{2} \wedge e_{6} \wedge e_{7}-\tau_{31}^{2} e_{3} \wedge e_{5} \wedge e_{7} \\
& +\cdots \\
& -\tau_{12} \Delta e_{1} \wedge e_{5} \wedge e_{6}-\tau_{13} \Delta e_{1} \wedge e_{5} \wedge e_{7}-\tau_{23} \Delta e_{1} \wedge e_{6} \wedge e_{7} \\
& -\left(\alpha_{1} \Delta+\tau_{12} \tau_{13}\right) e_{3} \wedge e_{5} \wedge e_{6}+\left(-\alpha_{1} \Delta+\tau_{12} \tau_{13}\right) e_{4} \wedge e_{5} \wedge e_{7} \\
& +\left(\alpha_{2} \Delta+\tau_{12} \tau_{23}\right) e_{2} \wedge e_{5} \wedge e_{6}+\left(-\alpha_{2} \Delta+\tau_{12} \tau_{23}\right) e_{4} \wedge e_{6} \wedge e_{7} \\
& +\left(\alpha_{3} \Delta+\tau_{13} \tau_{23}\right) e_{2} \wedge e_{5} \wedge e_{7}+\left(\alpha_{3} \Delta-\tau_{13} \tau_{23}\right) e_{3} \wedge e_{6} \wedge e_{7} \\
& -\Delta^{2} e_{5} \wedge e_{6} \wedge e_{7},
\end{aligned}
$$


where $\Delta=\alpha_{3} \tau_{12}+\alpha_{2} \tau_{31}+\alpha_{1} \tau_{23}$. Except for the last one above, all the other 34 coordinates are polynomials in $\alpha_{1}, \cdots, \tau_{31}$ of degree $\leq 3$. (We have displayed all the coordinates of degree 3 .)

Now we homogenize $W_{1} \wedge W_{2} \wedge W_{3}$ by (41). Hence these 35 coordinates are now sections of $\left(f^{-1} \mathcal{O}(1)\right)^{4}$. From this we see that the degree of the 2 nd associated curve of the directrix curve of a superminimal surface in $\mathbb{C} P^{34}$ is $4 \operatorname{deg}(f)$ minus the number of common zeros of these 35 sections.

In general consider a zero $q$ of $s_{0}$. Then all the coordinates, except possibly the last one above, are zero at $q$ after homogenization because all of them have a factor $s_{0}$.

If after homogenization the last coordinate is not zero at $q$, then $\left[W_{1} \wedge\right.$ $\left.W_{2} \wedge W_{3}\right]=\left[e_{5} \wedge e_{6} \wedge e_{7}\right]$ at $q$, which says that the 2 nd associated curve of the directrix curve passes through the point $\left[e_{5} \wedge e_{6} \wedge e_{7}\right]$ at $q$ in $\mathbb{C} P^{34}$, which in turn projects to the north pole in $S^{6}$, i.e., the underlying superminimal surface now passes through the north pole at $q$, violating our assumption. It then follows that $q$ is a zero of $\left(s_{0}\right)^{4} \Delta^{2}$; hence $q$ is a zero of the last coordinate with multiplicity at least 2 after homogenization. Let us look at other coordinates. If the degree of a coordinate is $\leq 2$ as a polynomial in $\alpha_{1}, \cdots, \tau_{31}$, then it has the factor $\left(s_{0}\right)^{2}$ after homogenization, and so $q$ is a zero of it with multiplicity at least 2 . When the coordinate is of degree 3 , say the one before last displayed above, then the second term $-\tau_{13} \tau_{23}$ of it has a factor $\left(s_{0}\right)^{2}$ after homogenization. On the other hand the first term of it is $s_{0} s_{3}\left(s_{0}^{2} \Delta\right)$ after homogenization, where $q$ is a zero of both $s_{0}$ and $s_{0}^{2} \Delta$. Therefore, $q$ is also a zero of this coordinate with multiplicity at least 2 after homogenization. In summary, for any zero $q$ of $s_{0}, q$ appears at least twice as a zero in all the 35 coordinates after homogenization. Now $s_{0}$ has $\operatorname{deg}(f)$ zeros so that we have at least $2 \operatorname{deg}(f)$ common zeros of these 35 sections of $\left(f^{-1} \mathcal{O}(1)\right)^{4}$.

We claim that these are all the possible common zeros. To see this, let $p$ be a common zero of these 35 sections other than the $2 \operatorname{deg}(f)$ zeros that we have found above. Since the first displayed coordinate of $W_{1} \wedge W_{2} \wedge W_{3}$ is $\left(s_{0}\right)^{4}$ after homogenization, $p$ is clearly a zero of $s_{0}$. Similarly, $p$ must be a zero of $s_{1}$ as well. For, look at the $\left(-\alpha_{1}^{2}\right)$-coordinate above, which is $-s_{0}^{2} s_{1}^{2}$ after homogenization. Since the $2 \operatorname{deg}(f)$ common zeros that we have removed all come from $s_{0}^{2}, p$ must then come from $s_{1}$. Continuing in this fashion, we see that $p$ is a common zero of $s_{0}, s_{1}, \cdots, s_{6}$, which violates the fact that $s_{0}, \cdots, s_{6}$ have no common zeros. This contradiction proves the claim. 
It follows from this claim that the degree of the 2nd associated curve of the directrix curve of a superminimal surface in $\mathbb{C} P^{34}$ is $4 \operatorname{deg}(f)-$ $2 \operatorname{deg}(f)=2 \operatorname{deg}(f)$, which completes the proof of the theorem.

Remark 2. The arguments in Theorem 2 give that in general the degree of the 2 nd associated curve of the directrix curve is $\geq 2 d$.

\section{$\S 5$. Existence of superminimal surfaces that are not almost complex}

We now prove the existence of superminimal surfaces, which are not almost complex, of arbitrarily large degrees on any Riemann surface $M$ of genus $g$. The following construction is a modification of the one in ([3]).

We choose to solve (7):

$$
\begin{aligned}
& d \sigma_{21}=-2 \alpha_{2} d \alpha_{1}, \\
& d \sigma_{23}=-2 \alpha_{2} d \alpha_{3}, \\
& d \sigma_{31}=-2 \alpha_{3} d \alpha_{1},
\end{aligned}
$$

subject to the constraint

$$
\tau_{23} \neq \sqrt{-1} \alpha_{1}
$$

Suppose we are given the meromorphic functions $\alpha_{1}, \sigma_{21}, \sigma_{31}$. Then $\alpha_{2}=-\frac{1}{2} d \sigma_{21} / d \alpha_{1}$ and $\alpha_{3}=-\frac{1}{2} d \sigma_{31} / d \alpha_{1}$. However, we have the constraint

$$
d \sigma_{23}=-\frac{1}{2} \frac{d^{2} \sigma_{31}}{d \alpha_{1}{ }^{2}} d \sigma_{21},
$$

which amounts to saying that the right hand side of the equation is an Abelian differential of the second kind with zero periods.

In the following, we refer to a divisor $D$ as being sufficiently positive if $\operatorname{deg}(D)$ is large enough so that $\operatorname{dim} H^{0}(D)=1-g+\operatorname{deg}(D) \gg 0$.

Fix a positive integer $s$ and pick $\alpha_{1}$ so that $\alpha_{1} \in \mathcal{L}(D)$ for some sufficiently positive effective divisor

$$
D=m_{1} P_{1}+\cdots+m_{s} P_{s}
$$

such that $\operatorname{deg}\left(\alpha_{1}\right)=\operatorname{deg}(D)$, so that the polar divisor of $\alpha_{1}$ is exactly $D$. Let the divisor of $d \alpha_{1}$ be

$$
\left(d \alpha_{1}\right)=n_{1} Q_{1}+\cdots+n_{t} Q_{t}-\left(m_{1}+1\right) P_{1}-\cdots-\left(m_{s}+1\right) P_{s}
$$


for some effective divisor $n_{1} Q_{1}+\cdots+n_{t} Q_{t}$. By replacing $\alpha_{1}$ by $\alpha_{1}+c$ for a suitable constant $c$, we may assume that

$$
R_{1}, \cdots, R_{\operatorname{deg}(D)}
$$

the zeros of $\alpha_{1}$, are all distinct and they are disjoint from all $Q_{j}$. Define the divisor

$$
D^{\prime}=a_{1} P_{1}+\cdots+a_{s} P_{s}-b_{1} Q_{1}-\cdots-b_{t} Q_{t}
$$

with $D^{\prime}$ sufficiently positive and $a_{i} \gg m_{i}, 1 \leq i \leq s, b_{j} \geq 2 n_{j}+2,1 \leq j \leq t$ so that we can pick $\sigma_{31}$ in $\mathcal{L}\left(D^{\prime}\right)$ in such a way that the polar divisor of $\sigma_{31}$ is $a_{1} P_{1}+\cdots+a_{s} P_{s}$. Then the divisor of $d^{2} \sigma_{31} / d \alpha_{1}^{2}$ is

$D_{0}+\left(b_{1}-2 n_{1}-2\right) Q_{1}+\cdots+\left(b_{t}-2 n_{t}-2\right) Q_{t}-\left(a_{1}-2 m_{1}\right) P_{1}-\cdots-\left(a_{s}-2 m_{s}\right) P_{s}$

for some effective divisor $D_{0}$. Finally we will now select $\sigma_{21}$ in $\mathcal{L}\left(D^{\prime \prime}\right)$ by choosing

$$
D^{\prime \prime}=c_{1} P_{1}+\cdots+c_{s} P_{s}+r P-d_{1} Q_{1}-\cdots-d_{t} Q_{t}
$$

to be some sufficiently positive divisor with $d_{j} \geq n_{j}+1$ for all $j$, where $P$ is neither in $D_{0}$ nor is it equal to any $P_{i}$ and $Q_{j}$, such that the right hand side of (43) will have zero periods. (Note that by our construction so far, the right hand side of (43) is already an Abelian differential of the second kind.) To justify this selection, observe that the period map

$$
u: f \longrightarrow\left(\int_{\gamma_{1}} \frac{d^{2} \sigma_{31}}{d \alpha_{1}^{2}} d f, \cdots, \int_{\gamma_{2 g}} \frac{d^{2} \sigma_{31}}{d \alpha_{1}^{2}} d f\right)
$$

where $\gamma_{1}, \cdots, \gamma_{2 g}$ generate $H_{1}(M, \mathbb{Z})$ is a linear map from $\mathcal{L}\left(D^{\prime \prime}\right)$ to $\mathbb{C}^{2 g}$. It is then clear that by letting $D^{\prime \prime}$ be sufficiently positive and $\operatorname{deg}\left(D^{\prime \prime}\right)$ sufficiently large the period map will have a nontrivial kernel of dimension at least $1-g+\operatorname{deg}\left(D^{\prime \prime}\right)-2 g=1-3 g+\operatorname{deg}\left(D^{\prime \prime}\right)$. On the other hand $\operatorname{dim} \mathcal{L}\left(D^{\prime \prime}-r P\right)=1-g+\operatorname{deg}\left(D^{\prime \prime}\right)-r$ if $D^{\prime \prime}-r P$ is sufficiently positive. Hence as long as $r>2 g$ we will be able to choose $\sigma_{21}$ with a pole at $P$ in this kernel. It is then easy to see by (8) that $d \tau_{23}$ has a pole at $P$ whereas $\alpha_{1}$ does not. In conclusion,

$$
\tau_{23} \neq \sqrt{-1} \alpha_{1}
$$

and so the corresponding superminimal surface is not almost complex in view of Proposition 6 . Since the the divisor of the holomorphic curve $C:=$ 
$\left[1: \alpha_{1}: \alpha_{2}: \alpha_{3}: \tau_{12}: \tau_{23}: \tau_{31}\right]$ can be chosen to be the least common multiple of the polar divisors of the six coordinate functions defining the curve, we see by Theorem 2, the remark following it and the construction in this section, that the degree of the constructed superminimal surface can be arbitrarily large.

We now show that the curve $C$ is of genus $g$, i.e., that the map $M$ to $C \subset \mathbb{C} P^{6}$ is generically one-to-one. Consider the evaluation map

$$
e: f \in \mathcal{L}\left(D^{\prime}\right) \longmapsto\left(f\left(R_{1}\right), \cdots, f\left(R_{\operatorname{deg}(D)}\right)\right) \in \mathbb{C}^{\operatorname{deg}(D)} .
$$

The kernel of $e$ is $\mathcal{L}\left(D^{\prime}-R_{1}-\cdots-R_{\operatorname{deg}(D)}\right)$, whose dimension is $1-g+$ $\operatorname{deg}\left(D^{\prime}\right)-\operatorname{deg}(D)$ if $\operatorname{deg}\left(D^{\prime}\right) \gg 0$. It then follows that $e$ is an onto map. We adjust the previous choice of $\sigma_{31}$ so that $e\left(\sigma_{31}\right)$ is a vector whose components are all distinct. In other words, the $\tau_{31}$-coordinate of $C$ assumes different values at the zeros of the $\alpha_{1}$-coordinate of $C$ because $\tau_{31}=\sigma_{31}+\alpha_{3} \alpha_{1}$. (Note that the image of $R_{1}, \cdots, R_{\operatorname{deg}(D)}$ on $C$ are affine points in $\mathbb{C} P^{6}$ by our construction.) Hence the map from $M$ to $C$ is generically one-to-one.

Lastly we show that the curve $C$ generates a linearly full superminimal surface in $S^{6}$.

LEMMA 1. A superminimal surface given by (42) is not linearly full if and only if there is a constant quadric vector $\left(w_{0}, z_{1}, z_{2}, z_{3}, w_{1}, w_{2}, w_{3}\right)$ satisfying (3) through (6).

Proof. The lemma is true because the 2-plane orthogonal to the 5-plane containing an $S^{4}$ in $S^{6}$ gives rise to the desired constant quadric vector, and vice versa.

Alternatively put, under the condition of the lemma, (3) through (6) are equivalent to

$$
\begin{aligned}
& w_{0}=\alpha_{1} z_{1}+\alpha_{2} z_{2}+\alpha_{3} z_{3}, \\
& w_{1}=-\alpha_{1} w_{0}+\tau_{12} z_{2}+\tau_{13} z_{3}, \\
& w_{2}=-\alpha_{2} w_{0}+\tau_{21} z_{1}+\tau_{23} z_{3}, \\
& w_{3}=-\alpha_{3} w_{0}+\tau_{31} z_{1}+\tau_{32} z_{2},
\end{aligned}
$$

which means that the curve $C$ lies in a projective subspace of $\mathbb{C} P^{6}$ cut out by these four linear equations. 
In view of Lemma 1 , it suffices to show that $1, \alpha_{1}, \alpha_{2}, \alpha_{3}$ are linearly independent for the generated superminimal surface to be linearly full in $S^{6}$, which is now easy to see by the above construction. In summary, we have established the following.

THEOREM 3. There exist linearly full superminimal surfaces, which are not almost complex, of genus $g$ and of any conformal structure with arbitrarily large degrees in $S^{6}$.

\section{$\S 6$. The superconformal case}

As mentioned in the introduction, all minimal surfaces in $S^{3}$ or $S^{4}$ and all almost complex surfaces in $S^{6}$ are either superminimal or superconformal. It is therefore desirable to understand the normalized potential explicitly in the superconformal case, which in principle determines such minimal surfaces.

Recall ([5]) that by the isotropic order of a harmonic map $f: M \longrightarrow$ $\mathbb{C} P^{n}$ we mean the maximal number $k$ for $k$ consecutive maps in the harmonic sequence of $f$ to be mutually orthogonal. In local terms, the harmonic sequence of $f$ are the projectivization of the local maps $e_{i}: M \longrightarrow$ $\mathbb{C}^{n+1},-\infty<i<\infty$, where $e_{0}$ is a local lift of $f$ and $e_{i+1}\left(e_{i-1}\right.$, respectively) is obtained from $e_{i}$ by taking $\partial$ ( $\bar{\partial}$, respectively) and projecting onto the linear space orthogonal to $e_{i}$. Note that a minimal surface in $\mathbb{C} P^{n}$ is superminimal precisely when one of the $e_{i}$ 's degenerates to zero. The isotropic order of a minimal surface in the sphere is its isotropic order when it is immersed in the complex projective space in the standard way.

Definition 3. A nonsuperminimal surface in $S^{n}$ is superconformal (or cyclic) if the isotropic order is $n$.

Without loss of generality we will work in $S^{2 n}$ from now on. Let $M$ be a simply connected region in $\mathbb{C}$ containing the origin and is parametrized by $z$. Since $f$ is real analytic ([13]), it suffices to consider the real analytic frames $F$ of $f$ and hence $\alpha_{0}$ and $\alpha_{1}$ in (12) are real analytic. Let $\alpha_{0}^{\prime}$ be the $(1,0)$-part of $\alpha_{0}$ and let $\beta_{0}(z)$ be the sum of the holomorphic terms, i.e., terms not involving $\bar{z}$, in the Taylor expansion of $\alpha_{0}^{\prime}(z)$ about 0 as a function of $z$ and $\bar{z}$. We will refer to $\beta_{0}$ as the holomorphic part of $\alpha_{0}^{\prime}$. Similarly, we denote by $\beta_{1}(z)$ the holomorphic part of $\alpha_{1}(z)$. The following is a simple and effective way of calculating the normalized potential ([28]). 
THEOREM 4. The normalized potential for $f$ with the origin as the reference point is given by

$$
P(z)=\Psi_{0}(z)^{-1} \beta_{1}(z) \Psi_{0}(z)
$$

where $\Psi_{0}$ is the solution to

$$
d \Psi_{0}=\beta_{0} \Psi_{0}, \quad \Psi_{0}(0)=I
$$

We will now apply this theorem to obtain the normalized potential for superconformal surfaces in $S^{2 n}$.

Consider the flag manifold $S O(2 n+1) / S O(2) \times \cdots \times S O(2)$, where $S O(2)$ appears $n$ times on the diagonal. With the $2 n$-involution

$$
\operatorname{diag}\left(1, \omega, \omega^{2}, \cdots, \cdots, \omega^{(n-1)},-1, \omega^{-1}, \cdots, \omega^{-(n-1)},-1\right)
$$

where $\omega$ is a $2 n$-th root of unity, $\mathcal{G}^{\mathbb{C}}=s o(2 n+1, \mathbb{C})$ admits an eigenspace decomposition as in (11). A superconformal surface in $S^{2 n}$ lifts to a primitive harmonic map in this flag manifold, and conversely, such a primitive map projects to a superconformal surface. To be explicit, for a superconformal surface $f$, one can exhibit an orthonormal basis $e_{0}, \cdots, e_{2 n}$ with

$$
E_{ \pm i}=\left(e_{2 i-1} \mp \sqrt{-1} e_{2 i}\right) / \sqrt{2}, \quad 1 \leq i \leq n
$$

such that $e_{0}$ is the position vector and

$$
\begin{aligned}
\partial_{z} e_{0} & =a_{1} E_{1} \\
\partial_{z}\left(E_{i}\right) & =a_{i+1} E_{i+1}+f_{i} E_{i}, \quad 1 \leq i \leq n-2, \\
\partial_{z}\left(E_{n-1}\right) & =a_{n} E_{n}+a_{-n} E_{-n}+f_{n-1} E_{n-1}, \\
\partial_{z}\left(E_{n}\right) & =-a_{-n} E_{-(n-1)}+f_{n} E_{n}, \\
\partial_{z}\left(E_{-n}\right) & =-a_{n} E_{-(n-1)}-f_{n} E_{-n}, \\
\partial_{z}\left(E_{-(i+1)}\right) & =-a_{i+1} E_{-i}-f_{i+1} E_{-(i+1)}, \quad 1 \leq i \leq n-2, \\
\partial_{z}\left(E_{-1}\right) & =-a_{1} e_{0}-f_{1} E_{-1},
\end{aligned}
$$

for a nonnegative real-valued function $a_{1}$ (the metric scalar), complexvalued functions $a_{i}$ 's depending on the frame whose absolute values are well-defined global functions, and some functions $f_{i}$ 's to be determined later. Hence for this special frame (it is unitary as well)

$$
F:=\left(e_{0}, E_{1}, \cdots, E_{n}, E_{-1}, \cdots E_{-n}\right)^{t r},
$$


we have

$$
\begin{aligned}
& \partial_{z}(F)=\left(A_{0}+A_{1}\right) F, \\
& \partial_{\bar{z}}(F)=-\left(A_{0}^{*}+A_{1}^{*}\right) F,
\end{aligned}
$$

with $A_{0} \in \mathcal{G}_{0}^{\mathbb{C}}$ and $A_{1} \in \mathcal{G}_{1}^{\mathbb{C}}$.

Moreover, up to a constant multiple,

$$
E:=\operatorname{tr}\left(A_{1}^{2 n}\right)=a_{1}^{2} a_{2}^{2} \cdots a_{n-1}^{2} a_{n} a_{-n}
$$

is a holomorphic function, which defines the Hopf differential $E(d z)^{2 n}$. Now set

$$
\begin{aligned}
\kappa_{i} & :=\left|a_{i}\right| / \sqrt{2} a_{1}, \quad 2 \leq i \leq n-1 \\
\kappa_{n} \pm \kappa_{-n} & :=\sqrt{2}\left|a_{ \pm n}\right| / a_{1} .
\end{aligned}
$$

The geometric meaning of these newly defined quantities is that attached to the superconformal surface is a direct sum of $n$ (real) 2-plane bundles $L_{i}$ spanned locally by $e_{2 i-1}, e_{2 i}, 1 \leq i \leq n . L_{1}$ is the tangent bundle of the surface with the first fundamental form $I(X, Y)$ whose ellipse of curvature is a circle of radius $2 a_{1}$, The second fundamental form $I I(X, Y)$ lives in $L_{2}$, where the ellipse of curvature is a circle with radius $\kappa_{2}$. The third fundamental form $I I I(X, Y, Z)=\left(\nabla_{Z} I I(X, Y)\right)^{\perp}$ is $L_{3}$-valued, whose ellipse of curvature is a circle of radius $\kappa_{3}$, etc., for $2 \leq i \leq n-1$. The ellipse of curvature of the $n$-th fundamental form assuming values in $L_{n}$ is no longer a circle in general, since the semi-major and minor axes assume lengths $2 \kappa_{n}$ and $2 \kappa_{-n}$, respectively. Note that the surface is superminimal precisely when one of $\kappa_{ \pm n}$ is zero.

Now it is straightforward to verify by employing the integrability condition (48) that we have

$$
f_{i}=\partial_{z} \log \left(a_{1} \overline{a_{2}} \cdots \overline{a_{i}}\right), \quad 1 \leq i \leq n
$$

It follows that

$$
A_{0}=\Psi_{z} \Psi^{-1}
$$

where

$$
\Psi=\operatorname{diag}(1, V, W)
$$

with $V$ being the vector

$$
\left(a_{1}, a_{1} \overline{a_{2}}, a_{1} \overline{a_{2} a_{3}}, \cdots, a_{1} \overline{a_{2} \cdots a_{n}}\right)
$$


and $W$ the vector each of whose components is the inverse of the corresponding component of $V$. Clearly, $A_{1}$ is the matrix whose entries are the $a_{i}$ 's in the appropriate slots given by the structural matrix in (46); we point out that the $(n-1,-n)$-slot is $a_{-n}$. A calculation shows $\Psi^{-1} A_{1} \Psi$ is the matrix in $\mathcal{G}_{\infty}^{\mathbb{C}}$ obtained by replacing $a_{i}$ in $A_{1}$ by $\left|a_{i}\right|^{2}, 1 \leq i \leq n$, and $a_{-n}$ by $E /\left.a_{1}^{4}\left|a_{2}\right|^{4}\left|a_{3}\right|^{4} \cdots\left|a_{n-1}\right|^{4}|| a_{n}\right|^{2}$. It is immediate now, in view of Theorem 4 , to write down the normalized potential of a superconformal surface.

THEOREM 5. Notation as above, let $\zeta_{i}(z)$ be the holomorphic part of $\left|a_{i}\right|^{2}, 1 \leq i \leq n$. With the standard frame (47) in $\mathbb{C}^{2 n+1}$ above, the normalized potential of a superconformal surface in $S^{2 n}$ is given by the matrix

$$
\left(\begin{array}{ccc}
0 & A & 0 \\
0 & B & C \\
-A^{t r} & 0 & -B^{t r}
\end{array}\right) d z
$$

where $A$ is the 1-by-n matrix $\left(\zeta_{1}, 0,0 \cdots, 0\right), B=\left(B_{i j}\right), 1 \leq i, j \leq n$, is the $n$-by-n matrix with $B_{i, i+1}=\zeta_{i+1}, 1 \leq i \leq n-1$, and zero elsewhere, and $C=\left(C_{i j}\right), 1 \leq i, j \leq n$, is skew-symmetric with $C_{n-1, n}=-C_{n, n-1}=$ $E / \zeta_{1}^{2} \cdots \zeta_{n-1}^{2} \zeta_{n}$ and zero elsewhere.

Note that when $E=0$, the potential in Theorem 5 reduces to the potential of a superminimal surface.

Indeed, in the superminimal case $a_{-n}=0$ and the directrix curve is $\left[E_{n}\right]$, and it is well-known that $\left|a_{n-i}\right|, 0 \leq i \leq n-1$, is exactly the metric scalar of the $i$-th and $(2 n-1-i)$-th associated curves of $\left[E_{-n}\right]$.

In general, for a holomorphic curve $f$ in $\mathbb{C} P^{N}$ with a local holomorphic lift $F$ into $\mathbb{C}^{N+1}$, the metric scalar of the curve is given, up to a constant multiple, by

$$
\partial_{z} \partial_{\bar{z}} \log \|F\|^{2}
$$

from which it is easy to see that the holomorphic part of the quantity in $(50)$ is

$$
\frac{d}{d z}\left(<F, F^{\prime}(0)>/<F, F(0)>\right)
$$

with $<,>$ the inner product of $\mathbb{C}^{N+1}$, which is simplified to

$$
\frac{d}{d z}\left(F_{1}(z) / F_{0}(z)\right)
$$

if $F_{i}(z)$ is the $i$-th coordinate of $F$ relative to a fixed unitary frame $\Lambda$ of $\mathbb{C}^{N+1}$ such that $\left(F(0), F^{\prime}(0), \cdots, F^{(n)}(0)\right)$ is $\Lambda$. 
With the various associated curves of $\left[E_{n}\right]$ understood to be $f$, it is not hard to see via (51) that we arrive at the potential (22) again.

\section{REFERENCES}

[1] J. Bolton, F. Pedit and L. Woodward, Minimal surfaces and the Toda field model, J. reine angew. Math., 459 (1995), 119-150.

[2] R. Bryant, Conformal and minimal immersions of compact surfaces into the 4-sphere, J. Differ. Geom., 17 (1982), 455-473.

[3] - Submanifolds and special structures on the octonians, J. Differ. Geom., 17 (1982), 185-232.

[4] Lie groups and twistor spaces, Duke Math. J., 52 (1985), 223-261.

[5] F. E. Burstall, Harmonic tori in spheres and complex projective spaces, J. reine angew. Math., 469 (1995), 149-177.

[6] E. Calabi, Isometric embeddings of complex manifolds, Ann. Math., 58 (1953), 1-23.

[7] — Minimal immersions of surfaces in Euclidean spheres, J. Differ. Geom., 1 (1967), 111-125.

[8] S. S. Chern, On the minimal immersions of the two sphere in a space of constant curvature, Problems in Analysis, Symposium in honor of Solomon Bochner, Princeton Univ. Press, Princeton, 1970, 27-49.

[9] Q. S. Chi and X. Mo, Rigidity of superminimal surfaces in complex projective spaces, Tôhoku Math. J., 44 (1992), 83-101.

[10] - The moduli space of superminimal surfaces of a fixed degree, genus and conformal structure in the four-sphere, Osaka J. Math, 33 (1996), 669-696.

[11] J. Dorfmeister, I. McIntosh, F. Pedit and H. Wu, On the meromorphic potential for a harmonic surface in a $k$-symmetric space, Manuscripta Math., 92 (1997), 143-152.

[12] J. Dorfmeister, F. Pedit and H. Wu, Weierstrass type representations of harmonic maps into symmetric spaces, Comm. Anal. and Geom., to appear.

[13] J. Eells and L. Lemaire, A report on harmonic maps, Bull. London Math. Soc., 10 (1978), 1-68.

[14] D. Ferus, F. Pedit, U. Pinkall and I. Sterling, Minimal tori in $S^{4}$, J. reine angew. Math., 429 (1992), 1-47.

[15] L. Fernandez, Superminimal surfaces in $S^{2 n}$, Thesis, Washington University, 1997.

[16] M. Guest and Y. Ohnita, Group actions and deformations for harmonic maps, J. Math. Soc. Japan, 45 (1993), 671-704.

[17] J. Hano, Conformal immersions of compact Riemann surfaces into the 2n-sphere $n \geq 2$, Nagoya Math. J., 141 (1996), 79-105.

[18] N.J. Hitchin, Harmonic maps from a 2-torus to the 3-sphere, J. Differ. Geom., 31 (1990), 627-710.

[19] D. Hoffman and W. Meeks, A complete embedded minimal surface with genus one, three ends and finite total curvature, J. Differ. Geom., 21 (1985), 109-127.

[20] D. Hoffman, F. S. Wei and H. Karcher, Adding handles to the helicoid, Bull. Amer. Math. Soc., 29 (1993), 77-84. 
[21] M. Kotani, Connectedness of the space of minimal 2-spheres in $S^{2 m}(1)$, Proc. Amer. Math. Soc., 120 (1994), 803-810.

[22] H. Karcher, U. Pinkall and I. Sterling, New minimal surfaces in $S^{3}$, J. Differ. Geom., 28 (1988), 169-185.

[23] B. Lawson, Complete minimal surfaces in $S^{3}$, Ann. Math., 92 (1970), 335-374.

[24] R. Miyaoka, The family of isometric superconformal harmonic maps and the affine Toda equations, J. Reine Angew. Math., 481 (1996), 1-25.

[25] R. Osserman, A Survey of Minimal Surfaces, Dover Publications, Inc., New York, 1986.

[26] F. S. Wei, Some existence and uniqueness theorems for doubly periodic minimal surfaces, Invent. Math., 109 (1992), 113-136.

[27] H. Wu, Banach manifolds of minimal surfaces in the 4-sphere, Amer. Math. Soc. Proc. Symp. Pure Math., 54 (1993), 513-539.

[28] A simple way for determining the normalized potentials for harmonic maps, Ann. Global Anal. Geom., 17 (1999), 189-199.

Quo-Shin Chi

Department of Mathematics

Washington University

St. Louis, MO 63130, U.S.A.

chi@math. wustl.edu

Luis Fernández

Department of Mathematics

Universidad de los Andes

Bogotá, Colombia

if ernand@uniandes.edu.co

Hongyou $\mathrm{Wu}$

Department of Mathematics

Northern Illinois University

DeKalb, IL 60115, U.S.A.

wu@math.niu.edu 